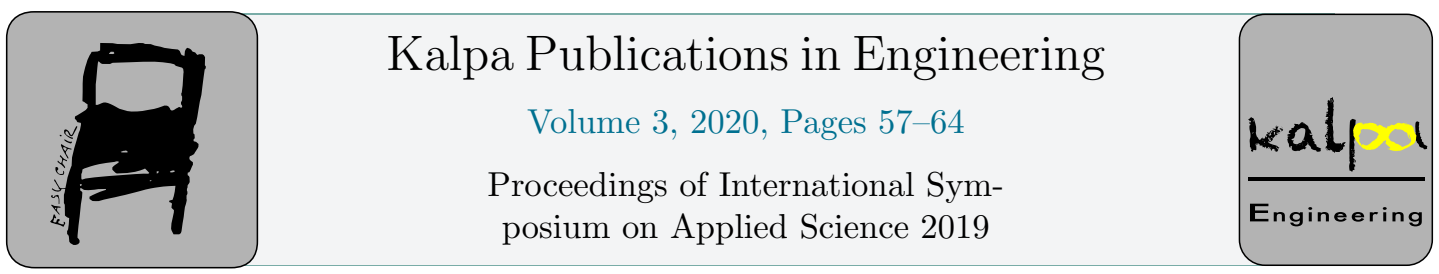

\title{
Assessment of the effectiveness of the NIR imaging systems applied to diagnose early dental lesions
}

\author{
Hai Mien Pham Thi ${ }^{1}$, Manh Cuong Hoang ${ }^{2}$, My Nhat Truong Thi ${ }^{1}$, Khac \\ Thinh Nguyen ${ }^{1}$, Phuoc An Truong Thi ${ }^{1}$ \\ ${ }^{1}$ Ho Chi Minh University of Technology, VNUHCM \\ ${ }^{2}$ University of Medicine and Pharmacy, Ho Chi Minh City \\ phamhaimien@hcmut.edu.vn
}

\begin{abstract}
In the recent years, oral problems are receiving more attentions from not only experts but also almost people. If tooth lesions in the early stages, such as white spots, hidden dental caries and tooth decay, etc., are not detected and treated promptly, they will lead to a risk of tooth loss. There are several common methods to detect tooth lesions such as clinical method, X-rays and particularly near infrared method, which is being developed significantly during the past decades. This method is substantially efficient for detecting early lesions by observing infrared images of tooth structures. In this study, near infrared (NIR) light at $850 \mathrm{~nm}$ was used for detecting dental lesions. Enamel is nearly translucent in NIR light, so the lesions located under enamel layer can be observed due to the interaction between NIR light and demineralized lesions. In addition, NIR method is not only a safe method but also able to detect dental lesions at the early stage and tooth cracks which X-rays are limited to detect. In dentistry, the accurate diagnosis requires a combination of methods. Nowadays, X-rays are not only the common method but also the gold standard in dentistry, so this study incorporated clinical evaluation and X-rays to make the criteria for dental lesion diagnosis. This article has aimed to analyze and evaluate the effectiveness of the NIR imaging systems in detecting dental lesions and compare NIR method to the clinical method combined with X-rays by Cohen's Kappa coefficient using SPSS (Statistical Package for the Social Sciences).
\end{abstract}

\section{Introduction}

Oral health, which is essential for a full health for everyone, has been given insufficient attention up to so far. As a result, tooth decay is affecting approximately $60-90 \%$ of children and adults. 
In dental diagnosis, dentists often use traditional methods such as clinical visual inspection or Xrays. In term of clinical observation, this is a visual method, although having some advantages like rapid and simple test, only is able to record significant cavities and ignores early lesions or hidden caries due to its low sensitivity. X-rays are the gold standard among diagnostic methods to determine the structural damage of teeth with high sensitivity in detecting dentin caries. Nevertheless, X-rays are limited in detection of early lesions and not suitable for regular monitoring because of the negative effect on one's heath by using ionizing radiation.

To be able to detect small, early and hidden lesions visually, not affecting the health of patients by a simple process, near infrared light is a tool that meets those requirements. Many scientific papers have demonstrated that near infrared wavelengths from $780 \mathrm{~nm}$ to $1550 \mathrm{~nm}$ are effective for detecting premature mineral loss under the occlusal and proximal surfaces [1]. On NIR images, the internal structure of teeth can be clearly observed as well as the damaged and healthy tissue are distinguished [2]. Therefore, the NIR technique is a potential method that can support X-rays for the diagnosis of precavities signs, limiting the level of regretful injury.

During the past years, scientists have mainly focused on comparing the effectiveness of NIR method with other classic methods such as Xrays or CT scan [3], [4] by using ROC graph, Cohen's Kappa coefficient by SPSS software (Statistical Package for the Social Sciences), etc. ROC graph is a common tool to evaluate the effectiveness of equipment which depends entirely on the X-rays gold standard. However, as above mentioned, $\mathrm{X}$-rays are not sensitive enough to detect some types of lesions, causing the inaccuracy of ROC graph. Therefore, the Kappa coefficient becomes the major tool to assess the similarity of diagnostic results of NIR method to other diagnostic methods. This article has aimed to analyze and evaluate the effectiveness of NIR method in detecting dental lesions by using transillumination and scattering techniques. The NIR diagnosis results were compared with those obtained by clinical method combined with X-rays according to Cohen's Kappa coefficient.

\section{MATERIALS AND METHODS}

\subsection{Sample collection and handling}

The assessment process of effectiveness of NIR method requires a large number of samples with different types of dental lesions. Tooth samples were collected regularly from many sources such as Odontology Department of National Hospital of Odonto-Stomatology, Odonto - Maxillo - Facial Hospital and An Binh Hospital - Odontology Department.

After being collected, tooth samples were cleansed of food, bacteria, blood and periodontal ligaments without losing the dental plaque and damaging the intrinsic lesions of samples. Each tooth sample had a separate base that was made of denture adhesive powder in order to keep the balance in the NIR and X-rays imaging. All the samples were preserved in physiological saline solution and $4 \%$ thymol in order to prevent samples from dehydrating during the study process as well as to ensure the sterilization.

\subsection{NIR systems for detection of dental lesions}

In order to observe lesions on occlusal surfaces of premolars and molars, a scattering system was used, whereas the transillumination system was useful to detect approximal lesions or caries of thin teeth such as incisors and canines. These systems, which were designed and built by our group, were described in detail in the other study [5].

The optical scattering system consists of two symmetrical 850-nm LEDs located next to the two opposite sides of the tooth and a NIR camera placed above the occlusal surface of tooth sample (Fig 1B). The distance between the camera and tooth was adjusted to suit each tooth sample in order to 
record the most qualified images. LEDs were put side by side with the tooth surface to eliminate the glare of NIR images caused by the light escaping from LEDs.

The transillumination system consists of an 850-nm LED placed on one side of tooth sample. On the other side of sample, the camera was placed on a coaxial line with LED and tooth sample to capture images (Figure 1A).

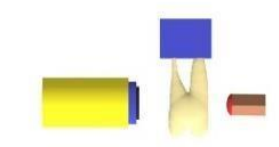

A.

Figure 1: Schematic diagrams of transillumination and scattering systems.

\subsection{Kappa evaluation method}

There are many statistical methods for assessment of the effectiveness of NIR method and in this study, Cohen's Kappa coefficient by the SPSS software was chosen. In medicine, Cohen's Kappa statistic is used to assess the degree of consensus between two judges in diagnosing a disease after removing random factors.

Because of the drawbacks of detecting early lesions by X-rays method as well as hidden or approximal caries by clinical examination, the diagnostic results on samples by $\mathrm{X}$-rays were associated with those obtained by clinical observation, providing the final conclusions of lesions (Table 1). After having the results of detection of dental lesions by the NIR method and clinical examination combined with X-rays method, these data were inserted into SPSS software to extract the Kappa coefficient for analyzing results [5] (Fig. 2). In regard to the import of data into the SPSS software, the positive results (samples with any type of lesions) were converted to 1 , the negative (healthy samples) - to 0 .

\begin{tabular}{|c|c|c|c|}
\hline \multirow{4}{*}{$\begin{array}{c}\text { Diagnostic } \\
\text { results }\end{array}$} & Clinical & X-rays & Conclusion \\
\cline { 2 - 4 } & Positive & Positive & Positive \\
\cline { 2 - 4 } & Pegative & Pogative & Positive \\
\cline { 2 - 4 } & Negative & Negative & Positive \\
\hline
\end{tabular}

Table 1: Combination of clinical observation with X-rays standards. 


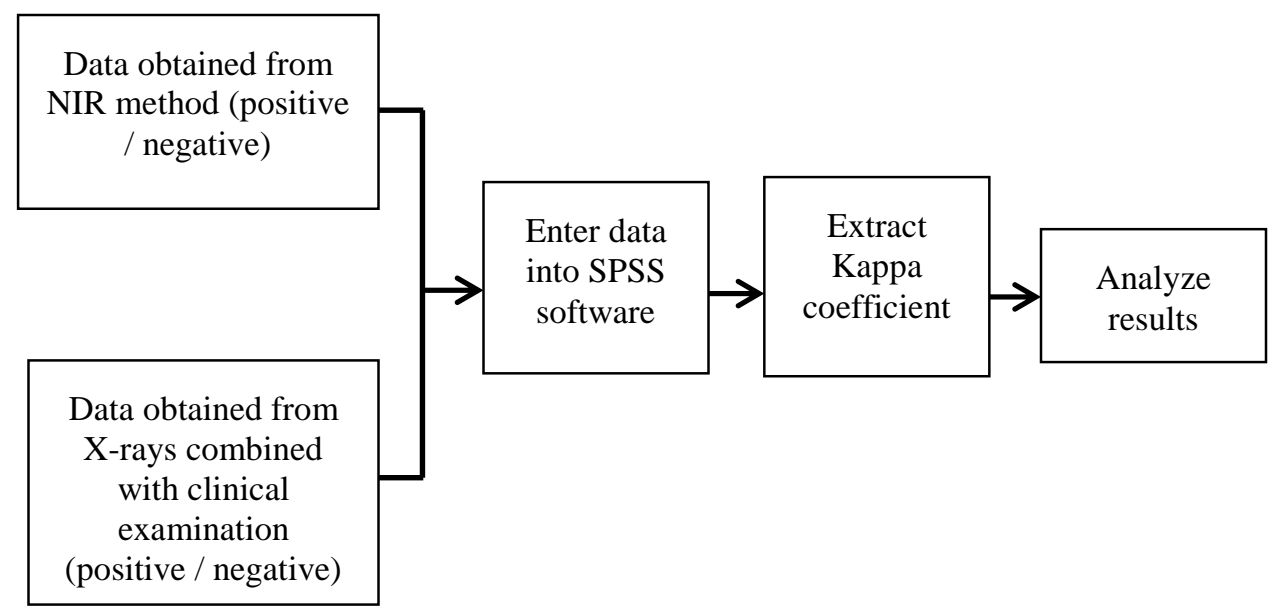

Figure 2: Assessment procesure on SPSS softwareAssessment procesure on SPSS software

\section{Results and discussion}

\subsection{NIR imaging results}

\section{A. Recurrent caries under fillings}

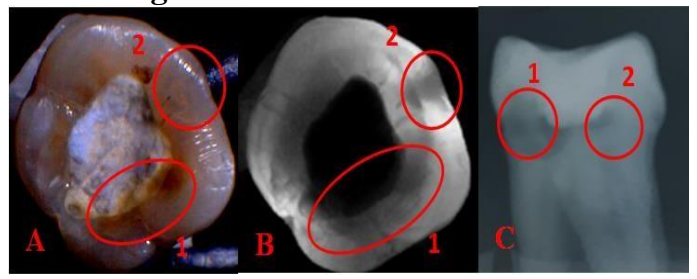

Figure 3: Sample 1 under visible light (A), NIR light (B), and X-rays

Tooth fillings are the way to restore damaged teeth to normal functions and shapes. In some cases, because of temperature changes, strong bite or incorrect filling technique, the gaps between tooth tissue and fillings can appear and be attacked by decay factors, causing recurrent caries under fillings.

Figure $3 \mathrm{~A}$ represents a tooth sample with a filling on the occlusal surface, which shows no signs of injury under visible light. However, in figure $3 \mathrm{~B}$, under infrared light, the enamel around the filling appears with some dark areas (circled areas). Also, the X-rays image (figure 3C) see the dark areas under the filling which indicate the existence of the recurrent caries under the filling.

It is worth to note that sound enamel has exceptionally low absorption and scattering coefficients in NIR wavelength regions [6], leading to the translucency of enamel on NIR images. In contrast, demineralized enamel has porous hollow structure of enamel crystals which increases the scattering in the teeth. Due to this multiple scattering, the NIR light is attenuated more by the absorption with the elongated path length in damaged tissue, which appears darker than surrounding healthy tissue.

The similar results of recurrent caries under the filling are depicted in Figures 4 - 5. This type of dental lesions is more difficult to be observed and diagnosed than others by clinical examination because lesion are corvered by fillings. 


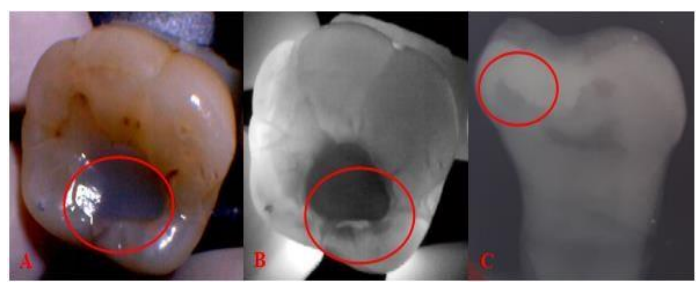

Figure 4: Sample 2 under visible light (A), NIR light (B), and X-rays (C).

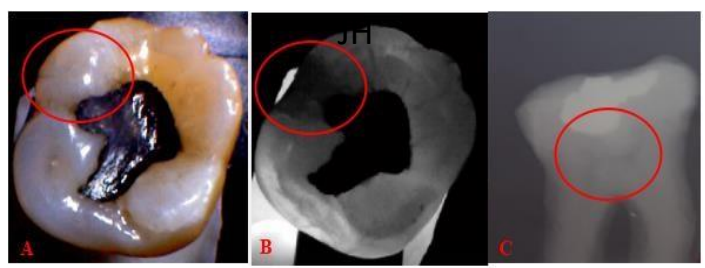

Figure 5: Sample 3 under visible light (A), NIR light (B), and X-rays (C).

\section{B. Hidden caries}

Hidden decay is a type of dental carries initially located under the enamel layer, no mark of which can be defined under visible light at the early stages. Figure 6 illustrates a tooth sample which contains the hidden caries. From figures $6 \mathrm{~A}-6 \mathrm{C}$ captured under visible light, it is clear that this sample is not seriously damaged, except the presence of plaque on the occlusal surface. However, NIR images (figures 6B) obtained by the scattering system indicate the appearance of a dark area located at the center of the plaque. To clarify this unusual sign, this sample was tested by the transillumination system and the results show that the darkened area continue to appear on NIR image (Figure 6D). Meanwhile, the X-ray film does not represent any lesions because of its poor contrast (Figure 7). Many studies have demonstrated that NIR method allows us to observe abnormalities within enamel due to the weak absorption and scattering of enamel in NIR region [7], providing a feasible tool to detect hidden lesions and interproximal lesions at the early stages without using X-rays.
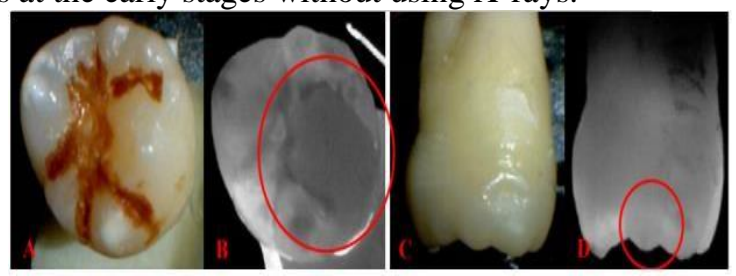

Figure 6: Sample 4 under visible light and NIR light: occlusal (A, B) and proximal (C, D).

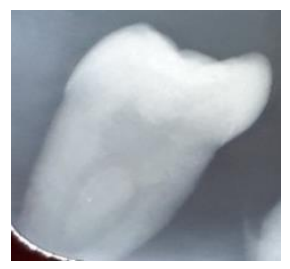

Figure 7: Sample 5 under X-rays. 


\section{White spot lesions}

White spot lesions are the result of demineralization in tooth enamel, manifested by a white mark on smooth surface of teeth in lowlevel danger. However, if white spot lesions are overlooked in clinical examination, they can lead to decay at the areas of mineral loss.

Figure 8A, which was captured under visible light, indicates the existence of a lot of white areas on the chewing surface of the sample, which are supposed to be either white spot lesions, or fluorosis or enamel opaque. Also, this is an extracted tooth sample, it is important to pay attention to the loss of water in tooth enamel which was able to cause the appearance of these white spots, which are apparent under visible light but have no signs on NIR images. In this case, under infrared light (Figure 8B), those white spots can not be observed clearly, with the exception of the smaller dark traces. Many studies have demonstrated that, under NIR light white spot lesions appear with high contrast because the scattering coefficient of dermineralized tooth tissue is lower than that of healthy enamel [8], whereas fluorosis or enamel opaque, do not affecting mineral density, behave as healthy enamel on infrared images. According the X-rays image (Figure 8C), this sample has positive status, because this method is unable to detect white spot lesions at early stages. The similar results of white spot lesions are depicted in figure 9.

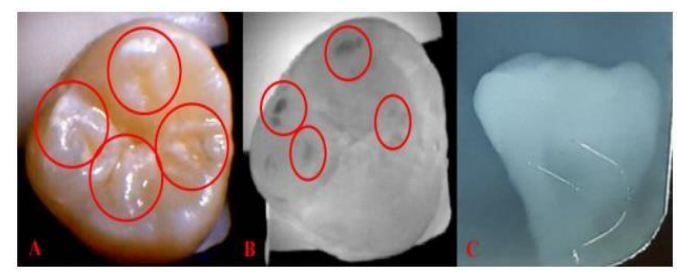

Figure 8: Sample 6 under visible light (A), NIR light (B), and X-rays (C).

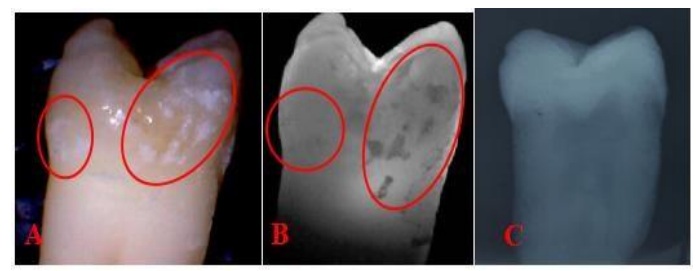

Figure 9: Sample 7 under visible light (A), NIR light (B), and X-rays (C).

\section{Cracked Teeth}

Cracks in teeth are caused by a variety of factors such as sudden temperature changes or powerful biting forces. A crack, which is not detected and treated properly, can create favorable conditions for bacteria to easily attack enamel and dentin. Figure 10 illustrates the surface of a tooth sample, which has no mark of lesions under visible light (Figure 10A), but appears in a dark trace under NIR light (figure 10B). The origin of this abnormal streak is that when NIR light, reaching the enamel, experiences multiple scattering within the crack and attenuated more by the absorption with the elongated path length. As a result, this area becomes darker than surroundings [9], [10]. This crack can be detected by using probe and magnifier but invisible in X-ray image (Figure 10C).

The results are similarly seen in tooth number 9 in Figure 11. 


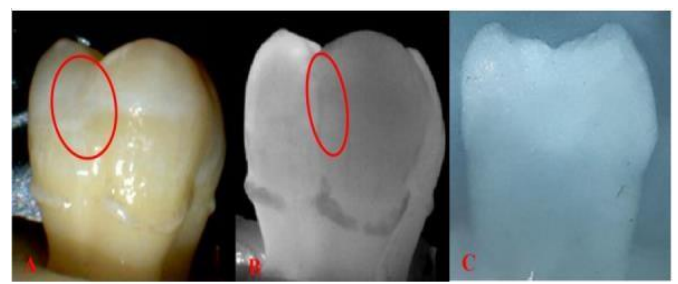

Figure 10: Sample 8 under visible light (A), NIR light (B), and X-rays (C).

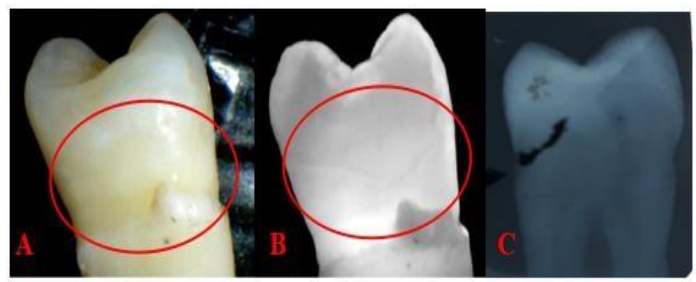

Figure 11: Sample 9 under visible light (A), NIR light (B), and X-rays (C).

\subsection{Evaluating the effectiveness of the NIR imaging systems}

Table 2 shows the total number of samples used in the statistics and their medical condition evaluated simultaneously by X-ray and NIR images. The positive diagnostic results (samples with any type of lesions) are represented by number 1 , the negative (healthy samples) - by number 0 . There are a total of 60 samples used in the experiment. The X-ray images combined with clinical examination demonstrate 47 positive samples (about $78.3 \%$ ) and 13 negative samples $(21.7 \%$ ). Among 13 negative samples according to X-rays method, there are 3 samples which have positive result by NIR images. Meanwhile, all positive samples by X-rays were simultaneously diagnosed with different types of lesions by NIR technique.

In this study, null hypothesis assumes that the NIR imaging systems and X-rays method combined with clinical observation provide the same diagnostic results. The results obtained from SPSS software (Table 2) indicate the very low p-value $(\mathrm{p}<0.005)$ and the high kappa coefficient $(\mathrm{k}=0.839)$ which demonstrate a high degree of similarity of diagnostic results by Xrays method and NIR technique.

\begin{tabular}{|l|c|c|c|c|}
\hline \multicolumn{2}{|c|}{} & \multicolumn{2}{|c|}{ IR } & \multirow{2}{*}{ Total } \\
\cline { 3 - 5 } \multicolumn{2}{|c|}{} & 0 & 1 & 13 \\
\hline \multirow{3}{*}{ X-Rays + Clinical } & 0 & 10 & 3 & 47 \\
\hline Total & 1 & 0 & 47 & 60 \\
\hline
\end{tabular}

Table 2. Summary of dental samples evaluated by clinical method combined with X-ray images and NIR method.

\begin{tabular}{|c|c|c|c|c|}
\hline & Value & Standard errors & $\begin{array}{c}\text { Approximate } \\
\text { value } \mathrm{T}^{\mathrm{b}}\end{array}$ & $\mathrm{p}^{\text {-value }}$ \\
\hline Kappa coefficient & 0.839 & 0.089 & 6.587 & $<0.0005$ \\
\hline Number of statistics N & 60 & & & \\
\hline
\end{tabular}




\section{Conclusion}

The study has analyzed the specific characteristics of some common types of tooth lesions such as white spot lesions, recurrent caries under fillings, hidden decay and tooth cracks. In order to analyze and evaluate NIR imaging systems applied to detect tooth lesions, the statistical report shows that our NIR imaging systems are as effective as the X-rays for detecting tooth lesions. In particular, NIR method has the higher sensitivity than X-rays in diagnosis of early lesions such as white spot lesions or cracked teeth. In addition, NIR method has many advantages of safety (not using ionizing radiation), real-time diagnosis, rapid test and flexible devices. Therefore, NIR method should be further researched and developed as an effective tool which is able to support clinicians to detect tooth lesions.

\section{Acknowledgment}

This research is funded by Ho Chi Minh City University of Technology - VNU-HCM, under grant number T-KHUD-2019-34.

\section{References}

Daniel Fried, Richard E. Glena, John D. B. Featherstone, Wolf Seka, "Nature of light scattering in dental enamel and dentin at visible and near-infrared wavelengts," Appl Opt, vol. 34, 1278-1285, (1995).

CL. Darling, GD. Huynh, D. Fried, "Light scattering properties of natural and artificially dermineralization dental enamel at 1310 nm," J Biomed Opt , 034023-1 - 034023-11, (2006).

J. Kühnisch, F. Söchtig, V. Pitchika, R. Laubender, K. W. Neuhaus, A. Lussi, R. Hickel, "In vitro validation of near-infrared transillumination at $780 \mathrm{~nm}$ for the detection of caries on proximal surfaces,"

Clinical Oral Investigations, DOI 10.1007/s00784-015-1559-4, (2015).

Chung Ng; E. C. Almaz, J. C. Simon, D. Fried, C. L. Darling, "Near-infrared imaging of demineralization on the occlusal surfaces of teeth without the interference of stains," J. of Biomediacal Optics, vol. 24, 036002, (2019).

Thi Hai Mien Pham, Phu Duong Le, Thi Kim Tham Ta, Attempting near-infrared transillumination imaging with simple instrumentation for studying dental lesions, Vietnam Journal of Science, Technology and Engineering, Vol.61(2), 42 - 46, (2019).

GD. Huynh, CL. Darling, D. Fried, "Changes in the optical properties of dental enamel at $1310 \mathrm{~nm}$ after demineralization," Lasers in dentistry X, Vols. 118-124, p. SPIE 5313, (2004).

Belinda Barton, Jennifer Peat, Medical Statistics A Guide to SPSS, Data Analysis and Critical Appraisal, John Wiley \& Sons Ltd, (2014).

Daniel Fried, Michal Staninec, Cynthia L. Darling (2010), "Near-infrared imaging of dental decay at 1,310 nm", J. Laser Dent., 18(1), 8-16.

F. Daniel, et al. (1995), "Nature of light scattering in dental enamel and dentin at visible and nearinfrared wavelengths", Applied Optics, 34(7), 1278-1285.

Soojeong Chung, Daniel Fried, Michal Staninec, and Cynthia L. Darling (2011), "Multispectral near-IR reflectance and transillumination imaging of teeth", Biomedical Optics Express, 2(10), 28042814. 